\title{
Structural Materials for Fusion Reactors
}

\author{
M. Victoria, N. Baluc and P. Spätig \\ EPFL-CRPP Fusion Technology Materials, CH-5232 Villigen PSI, Switzerland \\ e-mail: Philippe.Spatig@psi.ch
}

\begin{abstract}
In order to preserve the condition of an environmentally safe machine, present selection of materials for structural components of a fusion reactor is made not only on the basis of adequate mechanical properties, behavior under irradiation and compatibility with other materials and cooling media, but also on their radiological properties, i.e. activity, decay heat, radiotoxicity. These conditions strongly limit the number of materials available to a few families of alloys, generically known as low activation materials. We discuss the criteria for deciding on such materials, the alloys resulting from the application of the concept and the main issues and problems of their use in a fusion environment.
\end{abstract}

\section{Introduction}

The development of adequate structural materials is a major step towards fusion reactors becoming an efficient source of energy, particularly if the promise of an environmentally safe machine is to be maintained. The first wall, divertor, limiters and breeding blanket components are subjected not only to the high energy neutron environment resulting from the fusion reactions, but also to strong mechanical, heat and electromagnetic loadings. These conditions create a very severe operation frame for the materials used in these elements and have led to a long developmental path for the candidate materials.

The main idea in the materials development program to comply with the safety conditions is the development of low activation materials. The criteria for deciding on such materials, the resulting material families resulting from the application of the concept and the main issues and problems of their use in a fusion environment are discussed below.

\section{Effects on materials due to the fusion reactor environment.}

Eighty percent of the energy released by the D-T fusion reaction are transferred by $14 \mathrm{MeV}$ neutrons to the first wall and breeding blanket. The remaining $20 \%$ are carried by $\alpha$-particles issued from the same reaction, that together with other low energy neutral and charged particles will induce sputtering, erosion and blistering in the plasma facing materials.

About $10 \%$ of the energy of the $14 \mathrm{MeV}$ neutrons will be deposited in the first wall, the remaining energy being transferred mostly to the blanket [1]. Two types of radiation effects are produced in the materials:

(i) Inelastic $(n, x)$ interactions with nuclei which yield transmutation products and lead to the production of $\mathrm{He}, \mathrm{H}$ and other impurities in the bulk of the material.

(ii) The neutrons themselves plus the recoils resulting from the above nuclear reactions transfer energy to lattice atoms through elastic collisions and displace them from their normal sites. Through an iterative process, a displacement cascade is formed. About $10 \%$ of the vacancies and interstitial defects originally formed survive the evolution of the cascade and lead to the formation of a defect microstructure that hardens the material, to the formation of voids, to the redistribution of elements in the alloy inducing segregation and possibly to phase transformations.

The neutrons slow down as they penetrate the reactor structure, so the neutron spectrum changes with penetration depth. This will modify the recoil energy spectra as well as the transmutation rate, the magnitude of the radiation effects will be typically different in the first wall and blanket.

Radiation damage depends strongly on irradiation temperature $T_{\text {irr }}$ and three regions can be recognized in relation to the melting temperature of the material $\mathrm{T}_{\mathrm{M}}$ :

(i) At low temperatures $\left(\mathrm{T}_{\mathrm{ir}}<0.3 \mathrm{~T}_{\mathrm{M}}\right)$ the vacancies do not yet evaporate from their clusters. The microstructure is dominated by defect clusters and results in radiation hardening and a degradation of the fracture toughness (embrittlement) of the material, increasing its ductile to brittle transition temperature.

(ii) In the region of $0.3 \mathrm{~T}_{\text {irr }}<\mathrm{T}_{\text {irr }}<0.5 \mathrm{~T}_{\mathrm{M}}$, defects are strongly mobile and this results in phenomena such as radiation creep and swelling. 
(iii) At still higher temperatures, the presence of He leads to embrittlement through the formation of bubbles at grain boundaries.

All the above described effects limit the life of the components.

\section{Low activation materials}

While the burning of fission fuel produces long lived actinides, the fusion reaction does not intrinsically yield other radioactive elements. But fusion neutrons will activate the materials surrounding the plasma and this fact conditions the waste management and disposal scenarios. Under accident conditions, the decay heat is an important parameter, since heat enhances oxidation and possible volatilization and such release to the environment is the main contamination hazard in a loss of coolant condition [2].

In order to evaluate these parameters, extended cross section data libraries together with decay and activation data have been developed. In Europe, the present version of the FISPACT97/EAF97 [3,4] inventory code together with the decay and activation libraries EASY [5], which includes five reference fusion reactor neutron spectra (first wall, blanket, shield and two magnetic coils), have been used to show that only a few primary elements can be considered. They are $\mathrm{C}, \mathrm{Si}, \mathrm{Ti}, \mathrm{Fe}, \mathrm{Cr}$ and $\mathrm{V}$. Other elements, such as $\mathrm{W}$ can be used in limited quantities. Moreover, all of the evaluations made up to present show the importance of the presence of typical tramp impurities present in these metals, such as $\mathrm{Al}, \mathrm{Ni}, \mathrm{Ag}, \mathrm{Co}, \mathrm{Nb}$ and others, that are detrimental because of their poor radiological properties.

\section{Main issues in development of Low Activation Materials}

The most intensive development in the past fifteen years has been that of the ferritic-martensitic steels (Low activation steels or LAS), based on the 7-10CrWVTa composition and which has led to the production of large casts in Japan (F82H steel) and in Europe (the EUROFER97 steel). These steels have reasonable thermophysical properties and, from irradiation experience in fast reactors to well over $100 \mathrm{dpa}$ [6], a substantial resistance to swelling and high temperature embrittlement. Moreover, they have good compatibility with either water or He coolants and Libreeders. They are being developed for a temperature window up to $773 \mathrm{~K}$.

A critical issue in these steels is the existence of a ductile to brittle transition temperature (DBTT), which increases with irradiation to temperatures well above room temperature, making the steel unusable as a structural component. It has been shown that reducing the $\mathrm{Cr}$ content to less than $9 \%$ strongly decreases the sensitivity to this phenomena [7] and in Europe a number of LAS families (OPTIFER, OPTIMAX) have been developed on this basis. It has also been shown that a positive consequence of using pure materials and clean processing is that low DBTT temperatures are obtained in the unirradiated steel, which increase only moderately after irradiation. A typical data set is that obtained for the difference in DBTT, measured from Charpy tests, between the virgin and irradiated condition $(\triangle \mathrm{DBTT})$ in the F82H and OPTIMAX A LAS, shown in Fig. 1. In both cases, the DBTT is below room temperature after irradiation to 2.5 dpa.

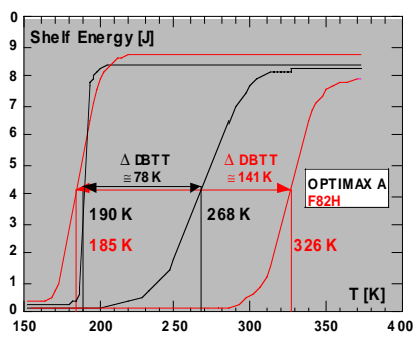

Figure 1:Shift of the DBTT for the F82H and OPTIMAX A steels after 2 dpa irradiation
There are a number of issues still open regarding the behavior of these LAS:

(i) Although the normal tensile properties are practically not affected, there is an increasing body of evidence showing that, contrary to what was expected in this temperature region, the presence of $\mathrm{He}$ induces an additional shift in the DBTT, already at low He contents [8]. There is a yet no clear unerstanding of what mechanism is operative

(ii) Although the actual reactor is expected to operate in a quasi-continuous mode, the design of intermediate or demonstration machines operates still in a pulsed fashion $(1000 \mathrm{~s}$ pulses in ITER). Such mode introduces mechanical damage through fatigue. As it is important to understand the actual dynamical situation under irradiation, which is nearer to the behavior of the material in the reactor, in-beam fatigue tests have been performed in the F82H steel under a 590 $\mathrm{MeV}$ proton beam irradiation [9]. 
(iii) The effects of ferromagnetism on plasma stability have to be investigated.

Titanium alloys have a number of properties that make them attractive structural material candidates for fusion reactors. High strength-to-weight ratio, intermediate strength values, good fatigue and creep rupture properties, small modulus of elasticity, high electrical resistivity, heat capacity, low coefficient of thermal expansion, low long-term ( $<10$ years after shutdown) residual radioactivity (after $\mathrm{V}$ and $\mathrm{Cr}$, Ti has the fastest decay rate), a high corrosion resistance together with good compatibility with coolants such as lithium, helium and water, high workability and good weldability and commercial availability with established mine and mill capacity are some of the favorable properties [10]. Because of the numerous current applications of titanium alloys in the aerospace and medical domains, there exists an extended properties database and industrial experience on these materials. Ti-alloys can be divided into three major classes determined by their phase constituency, they are referred to as the alpha $(\alpha)$, beta $(\beta)$ and alpha/beta $(\alpha / \beta)$ alloys, where the $\alpha$ phase is hcp and the $\beta$ phase is bcc. The alloying elements used in the titanium system can be divided into two classes according to which phase the element stabilizes. The substitutional $\alpha$ stabilizers are $\mathrm{Al}, \mathrm{Zr}$ and $\mathrm{Sn}$, while the $\beta$ stabilizers are $\mathrm{V}, \mathrm{Cr}, \mathrm{Mn}$, $\mathrm{Fe}, \mathrm{Co}, \mathrm{Ni}$ and Mo. Furthermore, the intermetallic TiAl has also been proposed in the Japanese program. Leguey et al. [11] have recently reported results after irradiation with $590 \mathrm{MeV}$ protons (mean dose rate: $3 \times 10^{-6} \mathrm{dpa}_{\mathrm{s}}^{-1}$; mean He production rate: $50 \mathrm{appm} / \mathrm{dpa}$ ) of pure Ti, for irradiation doses ranging between $10^{-3}$ and $10^{-1} \mathrm{dpa}$. Proton-irradiation at $330 \mathrm{~K}$ induces a significant increase in the critical stress measured at $0.2 \%$ plastic strain [11] and a decrease in the work hardening rate, as shown in Fig. 2. Hardening is of the order of about $40 \%$ per dpa for a dose of 0.03 dpa. It clearly relates to the irradiation-induced formation of a high density of defect clusters observed with transmission electron microscopy (TEM). About $20 \%$ of the observed defects were identified as dislocation loops with a mean apparent size of $5 \mathrm{~nm}$. No clear correlation could be established between ductility and irradiation dose, due to large scattering in the measurements. However, the total strain at fracture of all deformed specimens (irradiated or not) lies between 18 and $30 \%$. The TEM observation of specimens irradiated to $0.03 \mathrm{dpa}$ and deformed up to fracture shows the simultaneous annihilation of defect clusters as a result from interactions with mobile dislocations together with the formation of twins and dislocation cells. No void formation was observed. A comparison of the mechanical properties of the $\alpha-\mathrm{Ti}-5 \mathrm{Al}-2.4 \mathrm{Sn}$ and $\alpha+\beta$ Ti-6Al-4V alloys has been performed by Marmy et al. [12] also after irradiation with $590 \mathrm{MeV}$ protons. In the unirradiated alloys it was found that the resistance to tensile deformation of both alloys is very similar. The critical stress measured at $0.2 \%$ plastic strain, $\sigma_{0.2}$, has a mean value of about $800 \mathrm{MPa}$ at ambient temperature (that is about $40 \%$ higher than that exhibited by a $9 \mathrm{CrWV}$ low activation ferritic/martensitic steel) and $450 \mathrm{MPa}$ at $500^{\circ} \mathrm{C}$ (the same value as that of the ferritic/martensitic steel). Both alloys exhibit good ductility. The total elongation of the $\alpha+\beta$ alloy, between 15 and $20 \%$, is slightly higher than that of the $\alpha$ alloy, whatever the test temperature between 20 and $500^{\circ} \mathrm{C}$. After proton irradiation, see Fig. 3, hardening and loss of ductility are observed in both alloys. However, these phenomena are much stronger for the $\alpha+\beta$ alloy than for the $\alpha$ alloy. In fact, irradiation at 623 $\mathrm{K}$ affects the $\alpha+\beta$ alloy strongly, with an increase of about $40 \%$ in the yield strength and a 65 $\%$ decrease in tensile elongation, while the $\alpha$ alloy irradiated under the same conditions shows only very little hardening and about 3\% reduction in elongation. The embrittlement of the $\alpha+\beta$ alloy is probably associated with the presence of a radiation induced phase precipitation observed in TEM. Similar phase instability under irradiation in the Ti6Al4V has already been observed by a number of investigators. Probably the biggest disadvantage of titanium and its alloys in a fusion environment is their high chemical affinity with hydrogen which leads to hydrogen embrittlement and a expected large tritium inventory. Early work established that for impact embrittlement, a critical level of hydrogen concentration was required and that the onset of embrittlement was probably associated with precipitation of the hydride phase [13]. Subsequently, it has been shown that additions of aluminum raise the critical level of hydrogen for embrittlement and this was tentatively attributed to an increase in the solubility limit [14]. The hydrogen solubility in titanium was first estimated to be less than 100 wppm below $523 \mathrm{~K}$ [15]. In general, the alloys that consist primarily of $\alpha$ phase have a lower solubility for hydrogen than alloys that are primarily $\beta$ phase. In the case of $\alpha+\beta$ alloys, the solubility increases with increasing amounts of $\beta$ phase. The three major sources of hydrogen isotopes in a fusion reactor environment are [10]: hydrogen produced in the metal by neutron transmutation reactions, 
interactions with the deuterium and tritium (D-T) fuel in the plasma chamber and interactions with tritium in the breeding material.

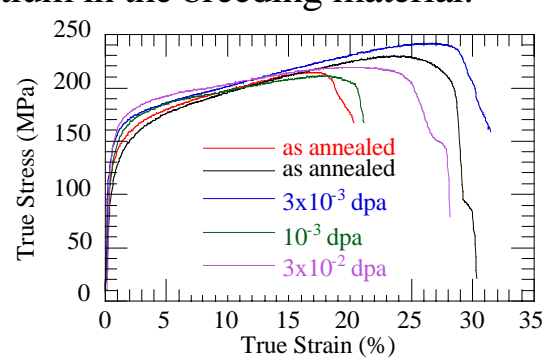

Figure 2: Tensile curve on pure Ti before and after proton irradiation

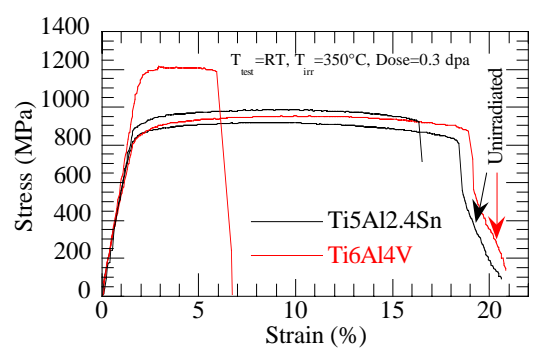

Figure 3: Tensile curve of Ti alloys, unirradiated condition and after 0.3 proton irradiation

Therefore, in order to make practical use of titanium alloys for fusion applications, hydrogen barrier coatings are necessary to prevent hydrogen intake and eventual embrittlement of the material. Preliminary attempts of depositing a graded coating with a $\mathrm{Cr}_{2} \mathrm{O}_{3}+(\mathrm{Si}$ or $\mathrm{Ti}) \mathrm{O}_{2}$ top layer on the Ti-5Al-2.4V $\alpha$-alloy have been recently performed at the Materials and Surface Department of SULZER Innotec AG. These coatings have been tested for structural integrity and hydrogen permeation and appear quite promising [16].

vanadium alloys are also considered [17] because of their low thermal expansion which coupled to a low elastic modulus leads to low thermal stresses and a high heat flux capability. Their compatibility with pure Li makes them a good choice for a liquid lithium coolant breeder blanket concept. The typical operating window of this design, from 623 to $1023 \mathrm{~K}$ and $1 \mathrm{MPa}$ pressure, requires high temperature strength and good creep properties, conditions that are fulfilled by the $\mathrm{V}-(4-5) \mathrm{Cr}-(4-5) \mathrm{Ti}$ [18]. The feasibility of fabricating components from the alloy is being demonstrated by its use in the DIII-D RDP, for which a $1200 \mathrm{~kg}$ cast has been produced and worked into rod, plate and sheet. Postirradiation results [18] after irradiation at temperatures below $600 \mathrm{~K}$ show strong radiation hardening and a reduction of the uniform strain to values below $1 \%$ and an upward shift of the in the DBTT of the order of $560 \mathrm{~K}$. The uniform irradiation increases at higher irradiation temperatures: is $6 \%$ after irradiation at $873 \mathrm{~K}$. A correlation can be made indicating that brittle fracture behavior occurs whenever the yield strength exceeds $700 \mathrm{M} \mathrm{Pa}$.

The development of electrically insulating walls for coolant channels is a critical issue for selfcooled liquid metal systems. Of the candidate coatings AlN deposited by vapor deposition have been shown unstable, while $\mathrm{CaO}$ coatings exhibit good electrical resistance during exposure to $\mathrm{Li}$ at $700 \mathrm{~K}$.

Fibre reinforced $\mathrm{SiC}$-SiC ceramic composites have gained strong interest in the fusion materials community due to their good low activation and decay heat properties at short and intermediate decay times, coupled to high mechanical strength for temperatures up to $1273 \mathrm{~K}$. Their microstructure consists of $\mathrm{SiC}$ embedded in a $\mathrm{SiC}$ matrix through a fiber-matrix interphase and are typically synthesized by the chemical vapor infiltration (CVI) process. They have a good compatibility with He, which makes them primary candidates for a high temperature, He cooled blanket. Early irradiation results [19] indicated a rapid degradation of the mechanical properties already at doses of $\approx 1 \mathrm{dpa}$, due to dimensional instability of the fiber and carbon interphase that leads to delamination. The present generation of $\mathrm{SiC}$ composites, with low oxygen, quasistoichiometric $\mathrm{SiC}$ fibers of enhanced crystalline perfection seem to have much improved properties after irradiation [20]. A number of critical issues are at present of concern:

(i) The relatively low thermal conductivity of $\mathrm{SiC}$ composites and the fact that it is further reduced by irradiation. This has already much improved in the materials produced with the new Hi Nicalon-S fibers in which a thermal conductivity value of the order of $50 \mathrm{~W} / \mathrm{mK}$ has been measured.

(ii) Void swelling in the temperature window of application.

(iii) The $(\mathrm{n}, \mathrm{x})$ cross-sections in $\mathrm{Si}$ are about one order of magnitude higher than for LAS or Valloys (about 150 appm $\mathrm{He}$ per dpa). The effects of this large amount of $\mathrm{He}$ at high temperatures is as yet unknown. 
(iv) The CVI process produces a microstructure that has about $10 \%$ porosity and is therefore permeable to gases.

(v) Technologically, methods for fabricating large components and of joining the composite to itself and to other metals need to be developed.

\section{Conclusions}

- In order to realize the potential safety and environmental advantages of fusion, low activation materials are being developed within a large international collaboration. The materials choice in this case is based not only on adequate mechanical properties, behavior under irradiation, and compatibility with other materials and cooling media, but also on their radiological properties.

- The alternative alloy classes being studied are ferritic-martensitic steels, V and Ti alloys and $\mathrm{SiC}-\mathrm{SiC}$ ceramic composites. At present, the ferritic-martensitic steels have achieved the greatest technological maturity.

- Common to all the alternatives is the lack of irradiation in a fusion relevant neutron environment, which limits our knowledge of the behavior of these materials in the proper reactor conditions (by example, on synergistic He effects on embrittlement, creep and swelling). Therefore, the availability of an adequate neutron source, such as the proposed International Fusion Materials Irradiation Facility (IFMIF) is of primary importance for the future development of the program.

\section{$\bullet$}

\section{Acknowledgements}

The present work has been supported by the European Fusion Technology Programme.

\section{References}

[1] D.R. Harries, "Ferritic Martensitic Steels for Use in Near Term and Commercial Fusion Reactors", Proceedings of the Topical Conference on Ferritic Alloys for Use in Nuclear Energy Technologies, Snowbird, Utah, June 19-23, 1983.

[2] K. Ehrlich, Phil. Trans. R. Soc. Lond. A 357 (1999) 595.

[3] D.R. Harries, G.J. Butterworth, A. Hishinuma, F.W. Wiffen, J. Nucl. Mater. 191-194 (1992) 92.

[4] R.A. Forrest, J.C. Sublet, FISPACT-97 User Manual, UKAEA FUS 358 Report, 1997.

[5] R.A. Forrest, J. Kopercky, "The European Activation System (EASY)", IAEA Advisory Group Meeting on FENDL-2, Vienna, November 1991.

[6] D.S. Gelles, J. Nucl. Mater. 233-237 (1996) 293.

[7] R.L. Klueh and G.J. Alexander, J. Nucl. Mater. 233-237 (1996) 336.

[8] R. Lindau, A. Möslang, D. Preininger, M. Rieth and H.D. Röhrig, "Influence of He on impact properties of reduced-activation ferritic-martensitic steels", Proc. Intern. Conf. On Fusion Reactor Mater. (ICFRM8) Sendai, Japan, October 1997.

[9] P. Marmy, "In beam fatigue properties of the F82H steel", Proc. Intern. Conf. On Fusion Reactor Mater. (ICFRM9), Colorado, USA, September 1999.

[10] J.W. Davis and D.L. Smith, J. Nucl. Mater. 85 \& 86 (1979) 71.

[11] T. Leguey, C. Bailat, N. Baluc and M. Victoria, to be submitted at the MRS Symposium on 'Microstructural Processes in Irradiated materials', Fall Meeting 2000.

[12] P. Marmy, Report on ITER TASK BL 14.2: Titanium Alloys Irradiation Testing (2000).

[13] C.M. Craighead, G.A. Lenning and R.I. Jaffee, Trans. AIME 194 (1952) 1317.

[14] L.W. Berger, D.N. Williams and R.I. Jaffee, Trans. AIME 212 (1958) 509.

[15] G.A. Lenning, C.M. Craighead and R.I. Jaffee, Trans. AIME 200 (1954) 367.

[16] T. Leguey, Private comunication.

[17] S.J. Zinkle, H. Matsui, D.L. Smith, A.F. Rowcliffe, E. van Osch, K. Abe and V.A. Kazakov, J. Nucl. Mater. 258-263 (1998) 205.

[18] B.A. Loomis and D.L. Smith, J. Nucl. Mater. 212-215 (1994) 799.

[19] P. Fenice, A.J. Frias Rebelo, R.H. Jones, A. Kohyama and L.L. Snead, J. Nucl. Mater. 258263 (1998) 215.

[20] A. Kohyama, this Conference. 